\title{
Association of Statin Use with Storage Lower Urinary Tract Symptoms: Data Mining of Claims Database
}

Mai Fujimoto, Tomoya Higuchi, Kouichi Hosomi and Mitsutaka Takada*

Division of Clinical Drug Informatics, School of Pharmacy, Kinki University, Japan

\begin{abstract}
Background:It remains uncertain whether or not statin use is associated with development of micturition disorders. To examine the association between statin use and the risk of storage lower urinary tract symptoms (LUTS), data mining was performed on a claims database.

Methods: Symmetry analysis was carried out to identify the risk of storage LUTS after statin use during the period from January 2005 to December 2011. Statin use in combination with drugs for storage LUTS was examined by prescription sequence symmetry analysis. Likewise, event sequence symmetry analysis was undertaken to evaluate the association between statin use and the diagnosis of storage LUTS and overactive bladder (OAB).

Results: Significant associations of statin use with drugs for storage LUTS were found, with an adjusted sequence ratio (ASR) of $1.58(1.08-2.33)$ at an interval of 6 months. In the analysis of drugs for OAB alone, significant associations were found, with ASRs of 1.82 (1.14-2.97) and 1.47 (1.06-2.04) at intervals of 6 and 12 months respectively. In analyses of individual statins and drugs for $\mathrm{OAB}$, a significant association was found for pravastatin, with an ASR of $2.66(1.15-6.88)$ at an interval of 6 months, but not for other statins. Significant associations were found for statin users in analyses of diagnosis of OAB, with ASRs of 2.00 (1.18-3.50) and 1.58 (1.10-2.28) at intervals of 6 and 12 months.

Conclusion: Analysis of the claims database demonstrated that statins might be associated with a new onset of storage LUTS. Statin-associated storage LUTS should be monitored closely in clinical practice, and further studies are needed to confirm our findings and elucidate the mechanism for statin-associated storage LUTS.
\end{abstract}

Keywords: Statin; Storage Lower Urinary Tract Symptoms (Storage LUTS); Symmetry analysis; Claims database

Abbreviations: LUTS: Lower Urinary Tract Symptoms; OAB: Overactive Bladder; ASR:Adjusted Sequence Ratio; PSSA: Prescription Sequence Symmetry Analysis; ESSA: Event Sequence Symmetry Analysis

\section{Introduction}

HMG-CoA reductase inhibitors (statins) are the most commonly prescribed cardiovascular drugs worldwide. The efficacy and safety of statins have been studied in a number of large trials of long duration $[1,2]$. A Europe-wide review of statins conducted by the Medicine and Healthcare Products Regulatory Agency (MHRA) assessed the evidence available on the following adverse reactions: sleep disturbances; memory loss; micturition disorders (problems with urination); sexual disturbances; depression; and interstitial pneumopathy with the use of statins (atorvastatin, fluvastatin, lovastatin, pravastatin, rosuvastatin and simvastatin) [3]. The MHRA Public Assessment Report evaluated the use of statins and the association between micturition disorders and problems with urination (micturition disorders, pollakiuria, nocturia, dysuria, micturition urgency, urinary retention, urinary incontinence, and oliguria) [3]. Consequently, the MHRA Public Assessment Report concluded that it was not considered necessary to list micturition disorder in the product information for the statins other than pravastatin, as the evidence for an association of micturition disorders with their use was weak [3]. However, it remains uncertain whether or not statin use is associated with development of micturition disorders.

In recent years, the term "micturition disorder" has been included in the classification of lower urinary tract symptoms (LUTS), which comprises voiding, storage, and postmicturition symptoms [4]. A population-based survey of a random sample of adult men and women conducted in 2005 in 5 countries found that $64.3 \%$ of adults reported at least one LUTS [5]. A large-scale multinational study revealed that $90 \%$ of men aged 50-80 years suffer from potentially troublesome LUTS [6]. Older age is a significant risk factor for LUTS[7-9]. Agerelated changes in the morphology and innervation of the detrusor muscle of the bladder and changes in bladder metabolism can cause storage LUTS [10]. The prevalence of overactive bladder (OAB), which is the representative disorder causing storage LUTS, was $16 \%-17 \%$ when measured in sample populations of adults in the United States [11] and six European countries [12]. Problems with urination are common in elderly patients; therefore statin-associated storage LUTS may go unnoticed in clinical practice, and it may not be easy to find an association between storage LUTS-related symptoms and drug therapy.

There are many conflicting reports concerning the association of statin use and the risk of storage LUTS [13-16]. Several studies have suggested that statin use over many years may be associated with a lower risk of developing LUTS. However, some studies reported that statins were not effective for alleviating LUTS [15], and another study revealed that statin use did not affect the development of LUTS in men [16]. In contrast, our previous study using the US Food and Drug

*Corresponding author: Mitsutaka Takada, PhD, Division of Clinical Drug Informatics, School of Pharmacy, Kinki University, 577-8502, 3-4-1, Kowakae, Higashi-osaka, Osaka, 577-8502, Japan, Tel: +81-6-6721-2332; Fax: +81-6-67301394; E-mail: takada@phar.kindai.ac.jp

Received September 16, 2014; Accepted October 10, 2014; Published October 20, 2014

Citation: Fujimoto M, Higuchi T, Hosomi K, Takada M (2014) Association of Statin Use with Storage Lower Urinary Tract Symptoms: Data Mining of Claims Database. J Pharmacovigilance 2: 147. doi:10.4172/2329-6887.1000147

Copyright: @ 2014 Fujimoto M, et al. This is an open-access article distributed under the terms of the Creative Commons Attribution License, which permits unrestricted use, distribution, and reproduction in any medium, provided the original author and source are credited. 
Administration (FDA) Adverse Event Reporting System (FAERS), which is the large spontaneous database of adverse event reports, demonstrated that statin use was associated with storage LUTS [13]. In addition, the analysis of a large database of prescriptions in Japan also demonstrated significant association of statin use with storage LUTS [17].

Recently, data mining with different methodologies and algorithms has been applied to identify safety signals within medical databases [18], including spontaneous adverse drug reaction databases, claims databases, and prescriptions databases. The aim of the study was to confirm the hypothesis that statin use is associated with storage LUTS using claims database.

\section{Materials and Methods}

\section{Data source}

A large and chronologically organized database of claims was utilized, which was constructed by a database vendor (The Japan Medical Data Center Co., Ltd, Tokyo, Japan [JMDC]) using standardized disease classifications and anonymous record linkage [19]. This database included about 1.2 million (approximately $1 \%$ of the population) cumulative insured persons, mainly company employees and their family members, from January 2005 to December 2011. Because most beneficiaries are working adults or their family members, the proportion of elderly patients aged $\geq 65$ years is low, and these elderly patients tend to be healthier than the general elderly population. The JMDC claims database consisted of monthly claims from medical institutions and pharmacies and included information about beneficiaries. The data included an encrypted personal identifier; age and gender of the patient; procedures and diagnosis codes according to the International Classification of Diseases, $10^{\text {th }}$ revision (ICD-10); and the name, dose, and number of days' supply of prescribed and/or dispensed drugs. All drugs were coded according to the Anatomical Therapeutic Chemical (ATC) classification of the European Pharmaceutical Market Research Association (EphMRA) [20]. An encrypted personal identifier can be used to link claims data from different hospitals, clinics, and pharmacies. For the prescription sequence symmetry analysis (PSSA), we utilized cases extracted from the JMDC claims database for which statins or drugs for $\mathrm{OAB}$ and neurogenic bladder were prescribed at least once during the study period [21]. In addition, cases diagnosed with storage LUTS and disorders causing storage LUTS were also extracted for the event sequence symmetry analysis (ESSA).

\section{Definition of LUTS or a disorder causing LUTS}

ICD-10 codes analyzed in the study are presented in Table 1. The LUTS classification comprises voiding symptoms (slow stream, splitting, spraying, intermittency, hesitancy, straining, and terminal dribble), storage symptoms (increased daytime frequency, nocturia, urgency, and urinary incontinence) and post-micturition symptoms [4]. Diagnoses in the JMDC claims database are classified by the ICD-10. The symptoms in the LUTS classification did not correspond correctly with the ICD-10 classification. Neurogenic bladder and OAB are the predominant disorders that may lead to the development of storage LUTS. The ICD-10 codes of N31 (Neuromuscular dysfunction of bladder) and N32.8 (Other specified disorders of bladder) were selected as neurogenic bladder and $\mathrm{OAB}$, respectively. In addition, the ICD-10 codes of N39.3, N39.4, R32, and R35 were selected as storage symptoms of LUTS. Other symptoms of LUTS could not be defined in the study.

\section{Symmetry analysis}

The current study used a retrospective design, applying the sequence symmetry method to evaluate asymmetry in the distribution of an incident event (e.g., either prescription of another drug or diagnosis) before and after the initiation of a specific treatment [22]. Asymmetry may indicate an association of the specific treatment of interest with the event. In this study, symmetry analysis was performed in two different ways. First, PSSA was performed to test the hypothesis that statin use may increase the risk for storage LUTS. The association of statin use with drugs for treatment of storage LUTS was analyzed. PSSA is based on the situation when drug $\mathrm{A}$ is suspected of causing an adverse event that itself is treated by drug B [22]. Patients were classified into two groups, "last" and "first" on the basis of the temporal order of the first recorded statins and the drug for LUTS prescriptions. The "last" group filled the number of patients prescribed drugs for storage LUTS after statin use, whereas conversely, "first" group filled the number of patients prescribed drugs for storage LUTS before statin use (Figure 1). In this study, drugs for the treatment of $\mathrm{OAB}$ and neurogenic bladder were used as markers of storage LUTS induced by statins. Next, ESSA was performed to evaluate the association between prescription of statins and diagnosis of storage LUTS and disorders causing storage LUTS.

The ratio of the number of persons with the event (either prescription of drugs for storage LUTS or diagnosis) after the initiation of statins versus the number of persons with the event before the initiation of statins are defined as the crude sequence ratio (SR). A SR $>1$ would be associated with an increased risk of statin-associated storage LUTS The SR is sensitive to prescribing or event trends over time. Therefore, SRs were adjusted for temporal trends in statins and drugs prescribed for storage LUTS or events using the method proposed by Hallas [22]. The probability for the statins to be prescribed first, in the absence of any causal relationship, can be estimated in a so-called null-effect SR $[22,23]$. The null-effect SR produced by the proposed model may be interpreted as a reference value for the SR. Thus, the null-effect SR is the expected SR in the absence of any causal association, taking the incidence trends into account. By dividing the crude SR by the nulleffect SR, an adjusted SR (ASR) can be obtained that is corrected for temporal trends. A slightly modified model was used to account for the limited time interval allowed between statin use and storage LUTS treatment [23]. The major advantage of the SR is that it is robust towards confounding factors that are stable over time.

\section{Data analysis}

The PSSA was undertaken to identify new use of solifenacin succinate, imidafenacin, tolterodine tartrate, mirabegron, propiverine hydrochloride, flavoxate hydrochloride, and oxybutynin hydrochloride as a surrogate for statin-associated storage LUTS or a disorder causing storage LUTS. Solifenacin succinate, imidafenacin, tolterodine tartrate, mirabegron, and propiverine hydrochloride are approved for $\mathrm{OAB}$, and

\begin{tabular}{|c|c|c|}
\hline ICD-10 code ${ }^{1}$ & Diagnosis & Definition in the study \\
\hline N39.3 & Stress incontinence & \multirow{4}{*}{ Storage symptom } \\
\hline N39.4 & Other specified urinary incontinence & \\
\hline R32 & Unspecified urinary incontinence & \\
\hline R35 & Polyuria & \\
\hline N32.8 & Other specified disorders of bladder & Overactive bladder \\
\hline N31 & $\begin{array}{l}\text { Neuromuscular dysfunction of } \\
\text { bladder, not elsewhere classified }\end{array}$ & Neurogenic bladder \\
\hline
\end{tabular}

${ }^{1}$ ICD-10: International Classification of Diseases, 10th Revision

Table 1: ICD-10 codes analyzed in the study. 


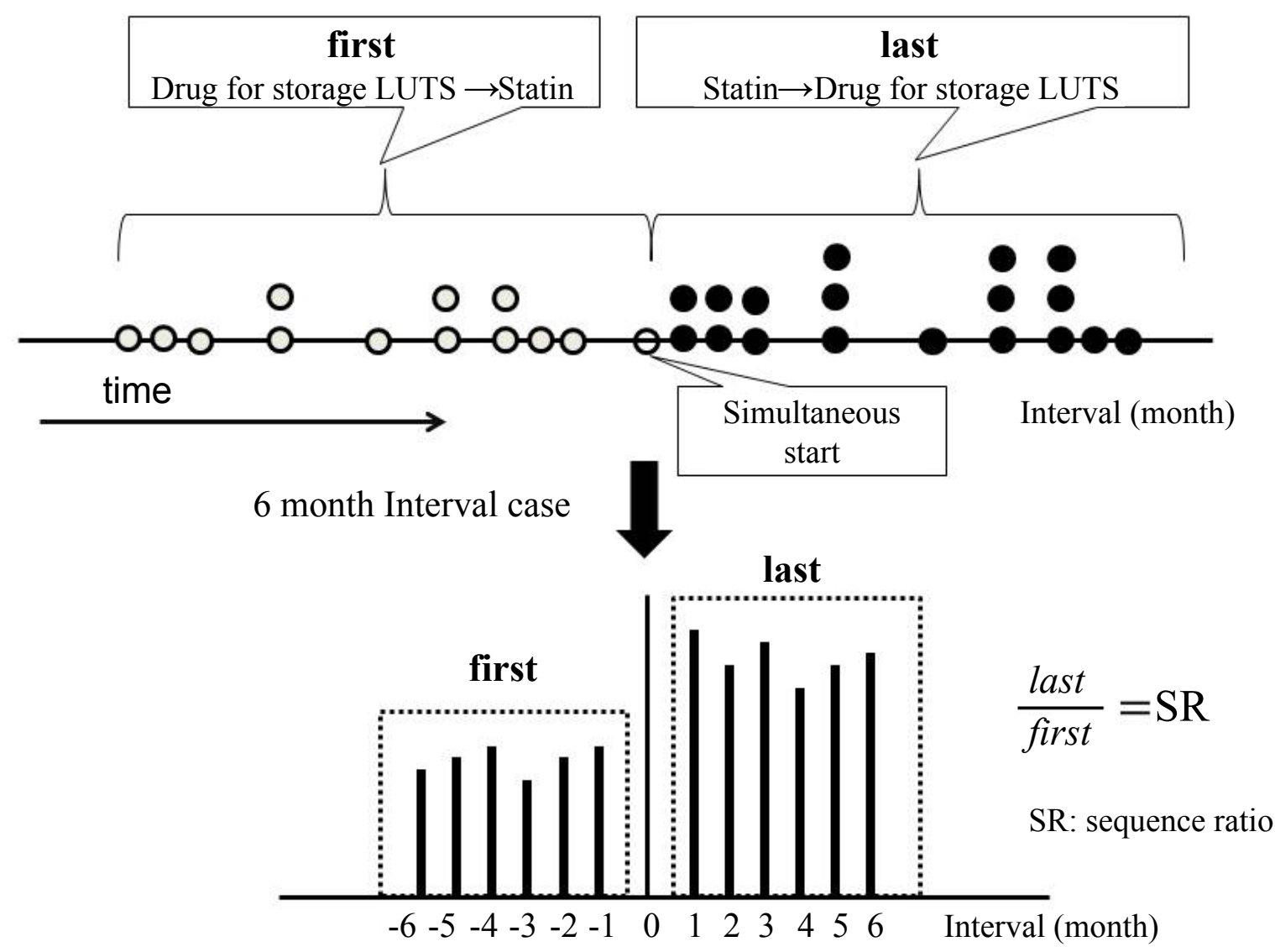

Figure 1: General picture of prescription sequence symmetry analysis.

Patients were classified into two groups, "first" and "last". By dividing the number of patients in group, "last" by the number of patients in group, "first", a crude sequence ratio (SR) can be obtained.

flavoxate hydrochloride and oxybutynin hydrochloride are approved for neurogenic bladder in Japan. These drugs are used for the treatment of storage LUTS, including frequency, urinary incontinence, urgency, and urge urinary incontinence. All incident users of statins and drugs for storage LUTS were identified during the period from January 2005 to December 2011. For this study, patients included in the database were followed up to December 2011. Therefore, different patients had different follow-up periods. Incidence was defined as the first prescription for target drugs. To exclude prevalent users of statins, the analysis was restricted to users who presented their first prescription in July 2005 or later; that is, after a run-in period of 6 months. To ensure that our analysis was restricted to incident users, we also carried out a waiting time distribution analysis [24]. An identical run-in period was also applicable to the case of patients enrolled into the cohort after June 2005 to exclude prevalent use of target drugs. The analysis was based on the principle that in observing first prescription occurrences within a specific time window, prevalent users of the drug will cluster at the beginning of the observation period as the prescribing is repeated within a short time period. In contrast, incident users will be evenly distributed throughout the observation period. Incident users were identified by excluding those individuals who had received their first prescription for the target drugs before July 2005. All patients who initiated a new treatment with statins and drugs for storage LUTS within 3-, 6-, and 12-month periods were identified. Relatively short intervals were employed to identify signals for events in the early stage of treatment. Patients who had received their first prescriptions for statins and drugs for storage LUTS in the same month were not included in the determination of SR.

Next, an ESSA was undertaken to identify individuals who had been diagnosed with statin-associated storage LUTS or disorders causing storage LUTS.All persons newly diagnosed with storage LUTS were identified during the period from January 2005 to December 2011. All incident users of statins and first diagnosis for storage LUTS or disorders causing storage LUTS were identified during the period from January 2005 to December 2011.

First prescriptions for statins and first diagnosis for storage LUTS or disorders causing storage LUTS should not have occurred in the same month. All patients who initiated a new treatment with statins and first diagnosis for storage LUTS or disorders causing storage LUTS within 3-, 6-, and 12-month periods were identified. Patients who had received their first prescriptions for statins and first diagnosis for storage LUTS or disorders causing storage LUTS in the same month were not included in the determination of SR. 95\% confidence intervals (95\% CI) for the ASRs were calculated using a method for exact confidence intervals for binomial distributions (Confidence Interval Analysis Software version 2.1.2, University of Southampton) [25]. The mean ages between males and females were compared using a Student's t-test.This study was approved by the Ethics Committee of Kinki University School of Pharmacy. 
Citation: Fujimoto M, Higuchi T, Hosomi K, Takada M (2014) Association of Statin Use with Storage Lower Urinary Tract Symptoms: Data Mining of Claims Database. J Pharmacovigilance 2: 147. doi:10.4172/2329-6887.1000147

Page 4 of 7

\begin{tabular}{|c|c|c|c|}
\hline & Total & Male & Female \\
\hline Users & 53,391 & & \\
\hline Receipts & $1,059,464$ & & \\
\hline Incident users, $n(\%)$ & 20,836 & $11,820(56.7)$ & $9,016(43.3)$ \\
\hline Age, years, $n(\%)$ & & & \\
\hline$<20$ & $45(0.22)$ & $22(0.19)$ & $23(0.26)$ \\
\hline $20-39$ & $2,772(13.3)$ & $2,207(18.7)$ & $565(6.30)$ \\
\hline $40-59$ & $13,249(63.6)$ & $7,417(62.8)$ & $5,832(64.7)$ \\
\hline $60-79$ & $4,689(22.5)$ & $2,166(18.3)$ & \\
\hline$\geq 80$ & $81(0.39)$ & $8(0.07)$ & \\
\hline Mean \pm SD & $51.8 \pm 10.7$ & $49.4 \pm 10.4$ & \\
\hline
\end{tabular}

1SD: Standard deviation

Table 2: Characteristics of the study population of statin users (January 2005 to December 2011).

\begin{tabular}{|c|c|c|c|c|c|c|c|c|c|c|}
\hline \multirow{2}{*}{$\begin{array}{l}\text { Statin incident users }{ }^{1} \text {, } \\
\qquad n=20,836\end{array}$} & \multirow{2}{*}{$\begin{array}{c}\text { Concomitant use } \\
\text { with therapeutic } \\
\text { drugs }\end{array}$} & \multirow{2}{*}{$\begin{array}{c}\text { Simultaneous } \\
\text { start }\end{array}$} & \multirow{2}{*}{$\begin{array}{l}\text { Interval } \\
\text { (months) }\end{array}$} & \multirow{2}{*}{\multicolumn{2}{|c|}{$\begin{array}{c}\text { No. of patients } \\
\text { prescribed drugs for } \\
\text { storage LUTS }^{2} \text { last/first }\end{array}$}} & \multirow{3}{*}{\begin{tabular}{|c|} 
Crude $\mathbf{S R}^{3}$ \\
1.32 \\
\end{tabular}} & \multirow{3}{*}{$\begin{array}{c}\begin{array}{c}\text { Null-Effect } \\
\text { SR }\end{array} \\
1.01\end{array}$} & \multirow{3}{*}{$\begin{array}{c}\text { Adjusted } \\
\text { SR } \\
1.31\end{array}$} & \multicolumn{2}{|c|}{$95 \% \mathrm{Cl}^{4}$} \\
\hline & & & & & & & & & Lower & Upper \\
\hline \multirow{3}{*}{ Drugs for storage LUTS } & \multirow{3}{*}{476} & \multirow{3}{*}{39} & 3 & 41 & 31 & & & & 0.80 & 2.16 \\
\hline & & & 6 & 75 & 47 & 1.60 & 1.01 & 1.58 & 1.08 & 2.33 \\
\hline & & & 12 & 124 & 98 & 1.27 & 0.99 & 1.27 & 0.97 & 1.68 \\
\hline \multirow{3}{*}{$\begin{array}{l}\text { Drugs approved for } \\
\text { treatment of } O A B^{6}\end{array}$} & \multirow{3}{*}{353} & \multirow{3}{*}{32} & 3 & 27 & 20 & 1.35 & 1.04 & 1.30 & 0.70 & 2.44 \\
\hline & & & 6 & 55 & 29 & 1.90 & 1.04 & 1.82 & 1.14 & 2.97 \\
\hline & & & 12 & 97 & 65 & 1.49 & 1.02 & 1.47 & 1.06 & 2.04 \\
\hline \multirow{3}{*}{ Solifenacin succinate } & \multirow{3}{*}{165} & \multirow{3}{*}{12} & 3 & 8 & 10 & 0.80 & 1.05 & 0.76 & 0.26 & 2.14 \\
\hline & & & 6 & 26 & 13 & 2.00 & 1.05 & 1.91 & 0.95 & 4.05 \\
\hline & & & 12 & 46 & 32 & 1.44 & 1.02 & 1.40 & 0.87 & 2.28 \\
\hline \multirow{3}{*}{ Imidafenacin } & \multirow{3}{*}{112} & \multirow{3}{*}{11} & 3 & 9 & 3 & 3.00 & 1.04 & 2.89 & 0.72 & 16.62 \\
\hline & & & 6 & 21 & 5 & 4.20 & 1.03 & 4.08 & 1.50 & 13.85 \\
\hline & & & 12 & 33 & 14 & 2.36 & 1.01 & 2.33 & 1.21 & 4.71 \\
\hline \multirow{3}{*}{ Tolterodine tartrate } & \multirow{3}{*}{28} & \multirow{3}{*}{1} & 3 & 8 & 0 & - & 1.06 & - & - & - \\
\hline & & & 6 & 8 & 0 & - & 1.07 & - & - & - \\
\hline & & & 12 & 12 & 1 & 12.00 & 1.04 & 11.55 & 1.71 & 493.56 \\
\hline \multirow{3}{*}{ Mirabegron } & \multirow{3}{*}{7} & \multirow{3}{*}{0} & 3 & 1 & 0 & - & 2.34 & - & - & - \\
\hline & & & 6 & 1 & 0 & - & 4.40 & - & - & - \\
\hline & & & 12 & 3 & 0 & - & 8.01 & - & - & - \\
\hline \multirow{3}{*}{$\begin{array}{l}\text { Propiverine } \\
\text { hydrochloride }\end{array}$} & & & 3 & 10 & 8 & 1.25 & 1.00 & 1.25 & 0.44 & 3.63 \\
\hline & 140 & 10 & 6 & 16 & 13 & 1.23 & 1.00 & 1.23 & 0.55 & 2.77 \\
\hline & & & 12 & 34 & 24 & 1.42 & 0.97 & 1.46 & 0.84 & 2.57 \\
\hline Drugs approved for & & & 3 & 15 & 13 & 1.15 & 0.97 & 1.19 & 0.53 & 2.71 \\
\hline treatment of disorders & 168 & 10 & 6 & 27 & 22 & 1.23 & 0.97 & 1.26 & 0.69 & 2.33 \\
\hline bladder & & & 12 & 41 & 41 & 1.00 & 0.97 & 1.03 & 0.65 & 1.63 \\
\hline & & & 3 & 12 & 10 & 1.20 & 0.97 & 1.23 & 0.49 & 3.19 \\
\hline Flavoxate hydrochloride & 122 & 5 & 6 & 19 & 18 & 1.06 & 0.98 & 1.08 & 0.54 & 2.18 \\
\hline & & & 12 & 28 & 35 & 0.80 & 0.98 & 0.82 & 0.48 & 1.38 \\
\hline & & & 3 & 4 & 3 & 1.33 & 0.97 & 1.37 & 0.23 & 9.35 \\
\hline hydrochloride & 57 & 6 & 6 & 11 & 5 & 2.20 & 0.95 & 2.30 & 0.74 & 8.46 \\
\hline & & & 12 & 17 & 9 & 1.89 & 0.94 & 2.02 & 0.85 & 5.14 \\
\hline
\end{tabular}

${ }^{1}$ Statin incident users: The number of patients received their first prescription for statins

'LUTS: Lower urinary tract symptoms

${ }^{3} \mathrm{SR}$ : Sequence ratio

${ }^{4} \mathrm{Cl}$ : Confidence interval

${ }^{5}$ No. of patients prescribed drugs for storage LUTS last: the number of patients prescribed drugs for storage LUTS after statin use

No. of patients prescribed drugs for storage LUTS first: the number of patients prescribed drugs for storage LUTS before statin use

${ }^{6} \mathrm{OAB}$ : Overactive bladder

Table 3: Symmetry analysis: Associations of statin use with drugs for storage LUTS.

\section{Results}

\section{Study population}

Characteristics of the study population are presented in Table 2 . The number of claims including statins during the study period was
$1,059,464$. Among the 53,391 statin users, 20,836 incident users were identified. The mean age of statin incident users was $51.8 \pm 10.7$ years.

Prescription sequence symmetry analysis (PSSA)

Associations of statins with drugs for storage LUTS are presented 


\begin{tabular}{|c|c|c|c|c|c|c|c|c|c|c|c|}
\hline & \multirow{2}{*}{$\begin{array}{c}\text { Incident } \\
\text { users }^{2}\end{array}$} & \multirow{2}{*}{$\begin{array}{c}\text { Concomitant use } \\
\text { with therapeutic } \\
\text { drugs }\end{array}$} & \multirow{2}{*}{$\begin{array}{c}\text { Simultaneous } \\
\text { start }\end{array}$} & \multirow{2}{*}{$\begin{array}{l}\text { Interval } \\
\text { (months) }\end{array}$} & \multirow{2}{*}{\multicolumn{2}{|c|}{$\begin{array}{c}\text { No. of patients } \\
\text { prescribed drugs for } \\
\text { OAB }^{3} \text { last } / \text { first }^{6}\end{array}$}} & \multirow{2}{*}{ Crude $\mathrm{SR}^{4}$} & \multirow{2}{*}{$\begin{array}{l}\text { Null-Effect } \\
\text { SR }\end{array}$} & \multirow{2}{*}{$\begin{array}{l}\text { Adjusted } \\
\text { SR }\end{array}$} & \multicolumn{2}{|c|}{$95 \% \mathrm{Cl}^{5}$} \\
\hline & & & & & & & & & & Lower & Upper \\
\hline \multirow{3}{*}{ Statins } & \multirow{3}{*}{20,836} & \multirow{3}{*}{353} & \multirow{3}{*}{32} & 3 & 27 & 20 & 1.35 & 1.04 & 1.30 & 0.70 & 2.44 \\
\hline & & & & 6 & 55 & 29 & 1.90 & 1.04 & 1.82 & 1.14 & 2.97 \\
\hline & & & & 12 & 97 & 65 & 1.49 & 1.02 & 1.47 & 1.06 & 2.04 \\
\hline \multirow{3}{*}{ Atorvastatin } & \multirow{3}{*}{7,241} & \multirow{3}{*}{147} & \multirow{3}{*}{9} & 3 & 9 & 9 & 1.00 & 1.03 & 0.97 & 0.34 & 2.76 \\
\hline & & & & 6 & 18 & 12 & 1.50 & 1.03 & 1.45 & 0.66 & 3.30 \\
\hline & & & & 12 & 38 & 28 & 1.36 & 1.01 & 1.35 & 0.80 & 2.28 \\
\hline \multirow{3}{*}{ Rosuvastatin } & \multirow{3}{*}{9,060} & \multirow{3}{*}{159} & \multirow{3}{*}{13} & 3 & 14 & 13 & 1.08 & 1.04 & 1.04 & 0.45 & 2.39 \\
\hline & & & & 6 & 26 & 20 & 1.30 & 1.03 & 1.26 & 0.68 & 2.38 \\
\hline & & & & 12 & 45 & 36 & 1.25 & 1.00 & 1.25 & 0.79 & 2.00 \\
\hline \multirow{3}{*}{ Fluvastatin } & \multirow{3}{*}{1,106} & \multirow{3}{*}{19} & \multirow{3}{*}{1} & 3 & 1 & 1 & 1.00 & 1.06 & 0.94 & 0.01 & 73.95 \\
\hline & & & & 6 & 2 & 1 & 2.00 & 1.09 & 1.83 & 0.10 & 108.27 \\
\hline & & & & 12 & 5 & 2 & 2.50 & 1.11 & 2.25 & 0.37 & 23.59 \\
\hline \multirow{3}{*}{ Pravastatin } & \multirow{3}{*}{5,782} & \multirow{3}{*}{99} & \multirow{3}{*}{9} & 3 & 9 & 4 & 2.25 & 1.07 & 2.11 & 0.59 & 9.37 \\
\hline & & & & 6 & 23 & 8 & 2.88 & 1.08 & 2.66 & 1.15 & 6.88 \\
\hline & & & & 12 & 28 & 13 & 2.15 & 1.09 & 1.98 & 0.99 & 4.17 \\
\hline \multirow{3}{*}{ Simvastatin } & \multirow{3}{*}{1,412} & \multirow{3}{*}{39} & & 3 & 5 & 2 & 2.50 & 1.05 & 2.39 & 0.39 & 25.06 \\
\hline & & & 5 & 6 & 7 & 4 & 1.75 & 1.06 & 1.64 & 0.42 & 7.66 \\
\hline & & & & 12 & 12 & 8 & 1.50 & 1.08 & 1.38 & 0.52 & 3.90 \\
\hline & & & & 3 & 7 & 7 & 1.00 & 1.03 & 0.97 & 0.29 & 3.24 \\
\hline Pitavastatin & 4,652 & 86 & 7 & 6 & 14 & 11 & 1.27 & 1.02 & 1.25 & 0.53 & 3.03 \\
\hline & & & & 12 & 24 & 19 & 1.26 & 0.99 & 1.28 & 0.67 & 2.47 \\
\hline
\end{tabular}

${ }^{1}$ Drugs approved for treatment of OAB comprise 5 drugs; solifenacin succinate, imidafenacin, tolterodine tartrate, mirabegron, and propiverine hydrochloride. ${ }^{2}$ Incident users: The number of patients received their first prescription for statin

${ }^{3} \mathrm{OAB}$ : Overactive bladder

${ }^{4} \mathrm{SR}$ : Sequence ratio

${ }^{5} \mathrm{Cl}$ : Confidence interval

${ }^{6}$ No. of patients prescribed drugs for OAB last: the number of patients prescribed drugs for OAB after statin use

No. of patients prescribed drugs for $O A B$ first: the number of patients prescribed drugs for OAB before statin use

Table 4: Symmetry analysis: Associations of individual statins with drugs approved for $O A B^{1}$.

in Table 3. Among the 20,836 statin incident users, 476 patients were identified as incident users of drugs for storage LUTS, before or after the initiation of statins. Significant associations of statins with drugs for storage LUTS were found, with an ASR of 1.58 (1.08-2.33) at an interval of 6 months. In the analysis of drugs approved for the treatment of $\mathrm{OAB}$ alone, significant associations were found, with ASRs of 1.82 (1.14$2.97)$ and 1.47 (1.06-2.04) at intervals of 6 and 12 months, respectively. In analyses of individual drugs, among the 20,836 statin incident users, $165,112,28,7,140,122$, and 57 patients were identified as incident users of solifenacin succinate, imidafenacin, tolterodine tartrate, mirabegron, propiverine hydrochloride, flavovate hydrochloride, and oxybutynin hydrochloride, before or after the initiation of statins, respectively. Significant associations were found for imidafenacin, with ASRs of 4.08 (1.50-13.85) and 2.33 (1.21-4.71) at intervals of 6 and 12 months, and for tolterodine tartrate, with an ASR of 11.55 (1.71-493.56) at an interval of 12 months. In analyses of individual statins and drugs approved for $\mathrm{OAB}$, a significant association was found for pravastatin, with an ASR of 2.66 (1.15-6.88) at an interval of 6 months, but not for other statins (Table 4).

\section{Event sequence symmetry analysis (ESSA)}

Associations of statins with diagnoses for storage LUTS and disorders causing storage LUTS are presented in Table 5. Among the 20,836 statin incident users, 281 cases were identified as incident cases with OAB. Significant associations were found for statin users, with ASRs of $2.00(1.18-3.50)$ and $1.58(1.10-2.28)$ at intervals of 6 and 12 months. Among the 20,836 statin incident users, 32, 15, 24, and 157 were identified as incident cases of stress incontinence (ICD-10: N39.3), other specified urinary incontinence (ICD-10: N39.4), unspecified urinary incontinence (ICD-10: R32), and polyuria (ICD-10: R35) before or after the initiation of statins, respectively. In additional analyses of individual statins and diagnoses, no significant association was found.

\section{Discussion}

Our results suggest that statin use may be associated with an increased risk of a new onset of storage LUTS. In the PSSA, a significant association was found between statins and drugs for storage LUTS. Drugs listed in Table 3 are approved for the treatment of storage LUTS induced by $\mathrm{OAB}$ or neurogenic bladder. In analyses of five drugs for $\mathrm{OAB}$, significant associations were found, but not for two drugs for neurogenic bladder. Furthermore, in the ESSA, a significant association with the ICD-10 code of N32.8 was found, but not with the ICD-10 code of N31. In the present study, ICD-10 codes of N31 (Neuromuscular dysfunction of bladder) and N32.8 (Other specified disorders of bladder) were defined as neurogenic bladder and $\mathrm{OAB}$, respectively, i.e., association of statins with $\mathrm{OAB}$ was found, but not with neurogenic bladder. Although neurogenic bladder is a disorder that causes storage LUTS, this disorder is associated with a specific causal mechanism including the underlying neurological disorder. It has been proposed that OAB is the leading cause of storage LUTS [4]. Our results might suggest that it is possible that the development of storage LUTS in statin users is associated with OAB induced by statin use. However, we could not obtain clear evidence that statin-associated storage LUTS was attributable to statin-induced OAB.

Although our study suggests that statin use may be associated with an increased risk of storage LUTS, there are many conflicting reports concerning this association. For example, several studies have suggested that statin use causes significantly less LUTS, demonstrating that statin use over many years may be associated with a lower risk of developing 


\begin{tabular}{|c|c|c|c|c|c|c|c|c|c|c|c|c|}
\hline \multirow{5}{*}{$\begin{array}{c}\begin{array}{c}\text { Statin incident } \\
\text { users } 1, n=20,836\end{array} \\
\text { Storage LUTS }\end{array}$} & \multirow{2}{*}{\multicolumn{2}{|c|}{ ICD-10 code $^{2}$}} & \multirow{5}{*}{$\begin{array}{c}\begin{array}{c}\text { Cases with } \\
\text { storage } \\
\text { symptoms }\end{array} \\
211\end{array}$} & \multirow{5}{*}{$\begin{array}{c}\begin{array}{c}\text { Simultaneous } \\
\text { start }\end{array} \\
19 \\
\end{array}$} & \multirow{3}{*}{$\begin{array}{c}\begin{array}{c}\text { Interval } \\
\text { (months) }\end{array} \\
3\end{array}$} & \multirow{2}{*}{\multicolumn{2}{|c|}{$\begin{array}{l}\text { Diagnosis } \\
\text { last/first }^{3}\end{array}$}} & \multirow{3}{*}{$\begin{array}{c}\begin{array}{c}\text { Crude } \\
\text { SR }^{4}\end{array} \\
1.15 \\
\end{array}$} & \multirow{3}{*}{$\begin{array}{c}\text { Null-Effect } \\
\text { SR } \\
1.00\end{array}$} & \multirow{3}{*}{$\begin{array}{c}\begin{array}{c}\text { Adjusted } \\
\text { SR }\end{array} \\
1.15\end{array}$} & \multicolumn{2}{|c|}{$95 \% \mathrm{Cl}^{5}$} \\
\hline & & & & & & & & & & & Lower & Upper \\
\hline & \multirow{3}{*}{\multicolumn{2}{|c|}{ N39.3, N39.4, R32, R35, }} & & & & 5 & 3 & & & & 0.51 & 2.63 \\
\hline & & & & & 6 & 28 & 19 & 1.47 & 1.01 & 1.46 & 0.79 & 2.77 \\
\hline & & & & & 12 & 57 & 42 & 1.36 & 1.02 & 1.33 & 0.88 & 2.04 \\
\hline \multirow{12}{*}{ Storage symptoms } & \multirow{3}{*}{ N39.3 } & \multirow{3}{*}{ Stress incontinence } & \multirow{3}{*}{32} & \multirow{3}{*}{4} & 3 & 1 & 2 & 0.50 & 0.99 & 0.51 & 0.01 & 9.75 \\
\hline & & & & & 6 & 2 & 4 & 0.50 & 0.97 & 0.52 & 0.05 & 3.60 \\
\hline & & & & & 12 & 6 & 8 & 0.75 & 0.97 & 0.76 & 0.22 & 2.49 \\
\hline & \multirow{3}{*}{ N39.4 } & \multirow{3}{*}{$\begin{array}{l}\text { Other specified urinary } \\
\text { incontinence }\end{array}$} & \multirow{3}{*}{15} & \multirow{3}{*}{0} & 3 & 0 & 1 & 0.00 & 1.06 & 0.00 & - & - \\
\hline & & & & & 6 & 1 & 3 & 0.33 & 1.06 & 0.31 & 0.01 & 3.91 \\
\hline & & & & & 12 & 5 & 4 & 1.25 & 1.08 & 1.16 & 0.25 & 5.55 \\
\hline & \multirow{3}{*}{ R32 } & \multirow{3}{*}{$\begin{array}{l}\text { Unspecified urinary } \\
\text { incontinence }\end{array}$} & \multirow{3}{*}{24} & \multirow{3}{*}{2} & 3 & 1 & 1 & 1.00 & 0.98 & 1.02 & 0.01 & 80.07 \\
\hline & & & & & 6 & 3 & 1 & 3.00 & 0.99 & 3.04 & 0.24 & 159.35 \\
\hline & & & & & 12 & 4 & 5 & 0.80 & 1.00 & 0.80 & 0.16 & 3.73 \\
\hline & \multirow{3}{*}{ R35 } & \multirow{3}{*}{ Polyuria } & \multirow{3}{*}{157} & \multirow{3}{*}{14} & 3 & 13 & 10 & 1.30 & 1.01 & 1.29 & 0.52 & 3.29 \\
\hline & & & & & 6 & 23 & 13 & 1.77 & 1.02 & 1.74 & 0.84 & 3.74 \\
\hline & & & & & 12 & 43 & 29 & 1.48 & 1.02 & 1.45 & 0.88 & 2.40 \\
\hline \multirow{3}{*}{ Overactive bladder } & \multirow{3}{*}{ N32.8 } & \multirow{3}{*}{$\begin{array}{c}\text { Other specified disorders of } \\
\text { bladder }\end{array}$} & & & 3 & 18 & 17 & 1.06 & 1.04 & 1.02 & 0.49 & 2.10 \\
\hline & & & 281 & 23 & 6 & 46 & 22 & 2.09 & 1.04 & 2.00 & 1.18 & 3.50 \\
\hline & & & & & 12 & 83 & 51 & 1.63 & 1.03 & 1.58 & 1.10 & 2.28 \\
\hline & & Neuromuscular dysfunction & & & 3 & 28 & 29 & 0.97 & 1.00 & 0.97 & 0.55 & 1.68 \\
\hline $\begin{array}{l}\text { Neurogenic } \\
\text { bladder }\end{array}$ & N31 & of bladder, not elsewhere & 268 & 17 & 6 & 47 & 41 & 1.15 & 1.01 & 1.14 & 0.73 & 1.78 \\
\hline & & & & & 12 & 72 & 57 & 1.26 & 1.00 & 1.26 & 0.88 & 1.82 \\
\hline
\end{tabular}

${ }^{1}$ Statin incident users; the number of patients received their first prescription for statins.

${ }^{2}$ ICD-10: International Classification of Diseases, 10th Revision

${ }^{3}$ Diagnosis last: the number of patients with diagnosis after statin use

Diagnosis first: the number of patients with diagnosis before statin use

${ }^{4} \mathrm{SR}$ : Sequence ratio

${ }^{5} \mathrm{Cl}$ : Confidence interval

${ }^{6}$ LUTS: Lower urinary tract symptoms

Table 5 Symmetry analysis: Associations of statin use with diagnoses.

LUTS [14]. Conversely, Mills et al. reported that atorvastatin was not effective for alleviating LUTS over a 6-month period in a randomized, double-blind, placebo-controlled clinical trial [15]. Stamatiou et al. also reported that lovastatin and finasteride did not change the development of LUTS, prostate volume, or prostate specific antigen concentrations in men with benign prostatic hyperplasia [16]. In contrast to those reports, our previous study using the FAERS database has demonstrated weak but reliable signals for LUTS in statin users [13]. Additionally, the analysis of the prescriptions database showed significant association for storage LUTS in statin users [26]. Although the mechanism responsible for these conflicting associations remains unknown, several noteworthy findings were reported. The Boston Area Community Health (BACH) Survey demonstrated an interesting finding that the use of statins is associated with a lower prevalence of urologic symptoms among older men but not among women or younger men. The observed associations among older men may be a manifestation of longer duration of use of statins [27]. Furthermore, St Sauver et al. reported that the longest duration of statin use was associated with the lowest risk of developing LUTS [14]. Our previous study using the FAERS database suggested that statin-induced LUTS might develop in the early stage of statin therapy [13]. Given these findings, the risk of LUTS might differ according to the duration of statin use.

The mechanisms of statin-associated storage LUTS are unknown. However, these adverse events may be attributable to reduction of cholesterol biosynthesis. Three possible mechanisms can be considered. First, normally, the serotonin signals are efficiently transmitted when receptors and other signal proteins are tied by a lipid raft [28]. Statins reduce production of cholesterol that is a major component of the lipid raft, leading to the impairment of serotonin neurotransmission [29]. This may cause affective disorders, which might cause storage LUTS via an autonomic imbalance. Secondly, statins inhibit brain cholesterol biosynthesis, with possible consequences for synaptic cholesterol homeostasis and myelin formation. Inadequate myelin production results in demyelination of central nervous system fibers, possibly leading to storage LUTS [30].Thirdly, Statins reduce the serum cholesterol level, which may affect the serum aldosterone level in our body [31]. Aldosterone's classical epithelial effect is to increase the transport of sodium across the cell in exchange for potassium and hydrogen ions, and is a major regulator of intravascular volume [32]. This situation may have the association with the cause of frequent urination. Assuming these mechanisms contribute to these reactions after statin use, it is possible that statin use increases the risk of storage LUTS.

There were several limitations associated with this study. First, our study population was selected from beneficiaries covered by the employees' health insurance system. Because most beneficiaries are working adults or their family members, the proportion of elderly patients aged $\geq 65$ years is low, and these elderly patients tend to be healthier than the general elderly population. Second, the insurance claims included a monthly summary of health care services provided by health care providers; thus, no date information was available for prescribing, dispensing, and diagnosis. Therefore, the month that a drug or diagnosis was first listed in the claim was defined as the initiation month for each, and when the initiation month of each prescription and event was the same, the two events were regarded as having simultaneous initiation. Third, the diagnoses listed in the claims were 
not validated. We generally needed to consider the diagnosis contained in the claims, which is listed for health insurance claims. Therefore, the patient may not actually have that disease. In addition, the defined symptoms for storage LUTS did not correspond correctly with the ICD10 classification. Fourth, a number of confounding factors associated with the development of storage LUTS and OAB should be investigated to ensure that our findings are reliable. Despite these limitations, significant associations of statin use with storage LUTS and OAB were identified in this study.

\section{Conclusion}

Analysis of the claims database demonstrated that statin therapy might be associated with a new onset of storage LUTS. Additionally, our study demonstrated that statin use was associated with diagnosis of $\mathrm{OAB} . \mathrm{OAB}$ is the representative disorder causing storage LUTS; therefore, it could be assumed that a positive association exists between statin-associated storage LUTS and OAB. Although the biological mechanism for this reaction remains unknown, the risk of storage LUTS associated with statin use is very important finding in clinical practice. These adverse events should be monitored closely in clinical practice, and further studies are needed to confirm our findings and elucidate the mechanism for statin-associated storage LUTS.

\section{Acknowledgement}

This study was done in the Division of Clinical Drug Informatics, School of Pharmacy, Kinki University, Higashi-osaka, Osaka, Japan.

We thank The Japan Medical Data Center Co., Ltd, Tokyo, Japan [JMDC] for providing the claims database.

\section{Conflict of Interest Statement}

No sources of funding were used to assist in the preparation of this study. Mai Fujimoto, Tomoya Higuchi, Kouichi Hosomi and Mitsutaka Takada have no conflicts of interest that are directly relevant to the content of this study.

\section{References}

1. Shepherd J, Cobbe SM, Ford I, Isles CG, Lorimer AR, et al. (1995) Prevention of coronary heart disease with pravastatin in men with hypercholesterolemia. West of Scotland Coronary Prevention Study Group. N Engl J Med 333: 1301 1307.

2. Taylor F, Huffman MD, Macedo AF, Moore TH, Burke M, et al. (2013) Statins for the primary prevention of cardiovascular disease. Cochrane Database Syst Rev 1: CD004816.

3. MHRA Public Assessment Report (2009) Statins: updates to product safety information.

4. Abrams P, Cardozo L, Fall M, Griffiths D, Rosier P, et al. (2002) The standardisation of terminology of lower urinary tract function: report from the Standardisation Sub-committee of the International Continence Society. Am J Obstet Gynecol 187: 116-126.

5. Irwin DE, Milsom I, Hunskaar S, Reilly K, Kopp Z, et al. (2006) Population-based survey of urinary incontinence, overactive bladder, and other lower urinary tract symptoms in five countries: results of the EPIC study. Eur Urol 50: 1306-1314.

6. Rosen R, Altwein J, Boyle P, Kirby RS, Lukacs B, et al. (2003) Lower urinary tract symptoms and male sexual dysfunction: the multinational survey of the aging male (MSAM-7). Eur Urol 44: 637-649.

7. Corcos J, Schick E (2004) Prevalence of overactive bladder and incontinence in Canada. Can J Urol 11: 2278-2284.

8. Yoshimura K, Terada N, Matsui Y, Terai A, Kinukawa N, et al. (2004) Prevalence of and risk factors for nocturia: Analysis of a health screening program. Int $\mathrm{J}$ Urol 11: 282-287.

9. Coyne KS, Zhou Z, Bhattacharyya SK, Thompson CL, Dhawan R, et al. (2003) The prevalence of nocturia and its effect on health-related quality of life and sleep in a community sample in the USA. BJU Int 92: 948-954.

10. Nordling $\mathrm{J}(2002)$ The aging bladder--a significant but underestimated role in the development of lower urinary tract symptoms. Exp Gerontol 37: 991-999.
11. Stewart WF, Van Rooyen JB, Cundiff GW, Abrams P, Herzog AR, et al. (2003) Prevalence and burden of overactive bladder in the United States. World J Urol 20: $327-336$.

12. Milsom I, Abrams P, Cardozo L, Roberts RG, Thüroff J, et al. (2001) How widespread are the symptoms of an overactive bladder and how are they managed? A population-based prevalence study. BJU Int 87: 760-766.

13. Fujimoto M, Hosomi K, Takada M (2014) Statin-associated lower urinary trac symptoms: data mining of the public version of the FDA adverse event reporting system, FAERS. Int J Clin Pharmacol Ther 52: 259-266.

14. St Sauver JL, Jacobsen SJ, Jacobson DJ, McGree ME, Girman CJ, et al (2011) Statin use and decreased risk of benign prostatic enlargement and lower urinary tract symptoms. BJU Int 107: 443-450.

15. Mills IW, Crossland A, Patel A, Ramonas H (2007) Atorvastatin treatment for men with lower urinary tract symptoms and benign prostatic enlargement. Eur Urol 52: 503-509.

16. Stamatiou KN, Zaglavira P, Skolarikos A, Sofras F (2008) The effects of lovastatin on conventional medical treatment of lower urinary tract symptoms with finasteride. Int Braz J Urol 34: 555-561.

17. Fujimoto M, Higuchi T, Hosomi K, Takada M (2014) Association of statin use with storage lower urinary tract symptoms (LUTS): data mining of prescription database. Int J Clin Pharmacol Ther 52: 762-769.

18. Takada M, Fujimoto M, Yamazaki K, Takamoto M, Hosomi K (2014) Association of statin use with sleep disturbances: data mining of a spontaneous reporting database and a prescription database. Drug Saf 37: 421-431.

19. Kimura S, Sato T, Ikeda S, Noda M, Nakayama T (2010) Development of a database of health insurance claims: standardization of disease classifications and anonymous record linkage. J Epidemiol 20: 413-419.

20. http://www.ephmra.org/

21. Hachiken H, Murai A, Wada K, Kuwahara T, Hosomi K, et al. (2013) Difference between the frequencies of antisecretory drug prescriptions in users of buffered vs. enteric-coated low-dose aspirin therapies. Int J Clin Pharmacol Ther 51 : 807-815.

22. Hallas J (1996) Evidence of depression provoked by cardiovascular medication a prescription sequence symmetry analysis. Epidemiology 7: 478-484.

23. Tsiropoulos I, Andersen M, Hallas J (2009) Adverse events with use of antiepileptic drugs: a prescription and event symmetry analysis. Pharmacoepidemiol Drug Saf 18: 483-491.

24. Hallas J, Gaist D, Bjerrum L (1997) The waiting time distribution as a graphica approach to epidemiologic measures of drug utilization. Epidemiology 8: 666670.

25. Morris JA, Gardner MJ (1988) Calculating confidence intervals for relative risks (odds ratios) and standardised ratios and rates. Br Med J (Clin Res Ed) 296 1313-1316.

26. Fujimoto M, Higuchi T, Hosomi K, Takada M (2014) Association of statin use with storage lower urinary tract symptoms (LUTS): data mining of prescription database. Int J Clin Pharmacol Ther 52: 762-769.

27. Hall SA, Chiu GR, Link CL, Steers WD, Kupelian V, et al. (2011) Are statin medications associated with lower urinary tract symptoms in men and women? Results from the Boston Area Community Health (BACH) Survey. Ann Epidemiol 21: 149-155

28. You H, Lu W, Zhao S, Hu Z, Zhang J (2013) The relationship between statins and depression: a review of the literature. Expert Opin Pharmacother 14: 14671476.

29. Tuccori M, Montagnani S, Mantarro S, Capogrosso-Sansone A, Ruggiero E, et al. (2014) Neuropsychiatric adverse events associated with statins: epidemiology, pathophysiology, prevention and management. CNS Drugs 28 : 249-272.

30. King DS, Wilburn AJ, Wofford MR, Harrell TK, Lindley BJ, et al. (2003) Cognitive impairment associated with atorvastatin and simvastatin. Pharmacotherapy 23 1663-1667.

31. Williams GH (2005) Aldosterone biosynthesis, regulation, and classical mechanism of action. Heart Fail Rev 10: 7-13

32. Bassett MH, White PC, Rainey WE (2004) The regulation of aldosterone synthase expression. Mol Cell Endocrinol 217: 67-74. 